\title{
BOLD Responses in Somatosensory Cortices Better Reflect Heat Sensation than Pain
}

\author{
Eric A. Moulton, ${ }^{1,2}$ Gautam Pendse, ${ }^{1,2}$ Lino R. Becerra, ${ }^{1,2}$ and David Borsook ${ }^{1,2}$ \\ ${ }^{1}$ Center for Pain and the Brain, Harvard Medical School, and ${ }^{2}$ Pain/Analgesia Imaging Neuroscience Group, Department of Psychiatry, Brain Imaging \\ Center, McLean Hospital, Harvard Medical School, Belmont, Massachusetts 02478
}

The discovery of cortical networks that participate in pain processing has led to the common generalization that blood oxygen leveldependent (BOLD) responses in these areas indicate the processing of pain. Physical stimuli have fundamental properties that elicit sensations distinguishable from pain, such as heat. We hypothesized that pain intensity coding may reflect the intensity coding of heat sensation during the presentation of thermal stimuli during fMRI. Six 3T fMRI heat scans were collected for 16 healthy subjects, corresponding to perceptual levels of "low innocuous heat," "moderate innocuous heat," "high innocuous heat," "low painful heat," "moderate painful heat," and "high painful heat" delivered by a contact thermode to the face. Subjects rated pain and heat intensity separately after each scan. A general linear model analysis detected different patterns of brain activation for the different phases of the biphasic response to heat. During high painful heat, the early phase was associated with significant anterior insula and anterior cingulate cortex activation. Persistent responses were detected in the right dorsolateral prefrontal cortex and inferior parietal lobule. Only the late phase showed significant correlations with perceptual ratings. Significant heat intensity correlated activation was identified in contralateral primary and secondary somatosensory cortices, motor cortex, and superior temporal lobe. These areas were significantly more related to heat ratings than pain. These results indicate that heat intensity is encoded by the somatosensory cortices, and that pain evaluation may either arise from multimodal evaluative processes, or is a distributed process.

\section{Introduction}

The sensation of pain rarely exists independently of other sensations, particularly when induced by contact heat. Heat and pain sensation are clearly separable percepts, as "how hot something feels" is distinctly different from "how much something hurts." Neuroimaging over the past decade suggests that pain intensity coding occurs over a network of cortical areas that includes the primary and secondary somatosensory cortex, anterior and posterior insula, and the anterior cingulate cortex (Derbyshire et al., 1997; Bushnell et al., 1999; Coghill et al., 1999; Bornhövd et al., 2002; Büchel et al., 2002; Porro et al., 2003; Ringler et al., 2003; Moulton et al., 2005; Johnstone et al., 2012). Such studies have used finely calibrated noxious stimuli, often in the form of heat, to evoke increasing levels of pain accompanied by increasing levels of brain activity. However, graded neural responses to a range of noxious temperatures may simply reflect intensity coding of a restricted range of heat sensation rather than pain per se.

Received Jan. 2, 2012; revised Feb. 9, 2012; accepted March 10, 2012.

Author contributions: E.A.M. designed research; E.A.M. performed research; E.A.M., G.P., and L.B. analyzed data; E.A.M., G.P., L.B., and D.B. wrote the paper.

This work was supported by the National Institutes of Health (National Institute on Drug Abuse Grant K01DA024289 to E.A.M.; and National Institute of Neurological Disorders and Stroke Grant K24NS064050 to D.B.). We acknowledge Jaime Knudsen, Lauren Nutile, and James Bishop for their help with subject recruitment and data collection.

The authors declare no competing financial interests.

Correspondence should be addressed to Dr. E. Moulton, Department of Psychiatry, Harvard Medical School,

P.A.I.N. Group, Brain Imaging Center, McLean Hospital, 115 Mill Street, Belmont, MA 02478. E-mail: emoulton@mclean.harvard.edu.

DOI:10.1523/JNEUROSCI.0006-12.2012

Copyright $\odot 2012$ the authors $\quad 0270-6474 / 12 / 326024-08 \$ 15.00 / 0$
To our knowledge, the possibility that the encoding of heat intensity may be misidentified as pain intensity has not been addressed in pain imaging. While highly correlated at higher temperatures, the percepts of heat and pain are clearly dissociable in the non-noxious range. Neuroimaging studies of innocuous heat-intensity coding indicate at least some degree of overlap with areas related to pain intensity, such as the insula (Craig et al., 2000; Olausson et al., 2005). This possibility suggests that the inclusion of more than one innocuous stimulus intensity level could better assess the specificity of heat versus pain intensity coding in the brain.

Though noxious and innocuous heat responses are differentiable by their temporal profile, pain intensity coding has rarely been considered in the context of the shape of the hemodynamic response. Previous studies reported that in many cases, the BOLD response to painful contact heat is biphasic, whereas the response to innocuous heat is monophasic (Becerra et al., 2001; Chen et al., 2002; Wager et al., 2004; Moulton et al., 2005; Upadhyay et al., 2010). The biphasic response pattern for noxious contact heat has been proposed to represent different circuit involvement for salience/threat detection (early phase) and the appraisal of pain intensity (late phase) (Becerra et al., 2001). The late phase of the biphasic response in primary somatosensory cortex has been reported to be capable of differentiating noxious heat stimuli separated by $1^{\circ} \mathrm{C}$ (Moulton et al., 2005). Considering the separate phases of the BOLD response to contact heat stimuli may also help differentiate pain versus heat-intensity coding in the brain.

We hypothesized that BOLD signals in brain areas responsive to heat are more reflective of perceived heat intensity than pain 
"High painful heat"

S1 Trial Average

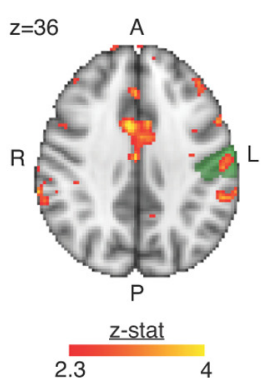

HRF Best Fit

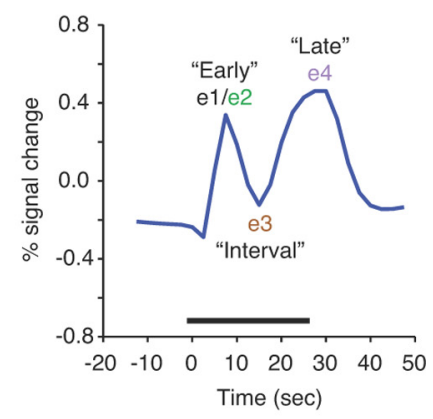

\section{Convolved EVs $\quad$ Unconvolved EVs}
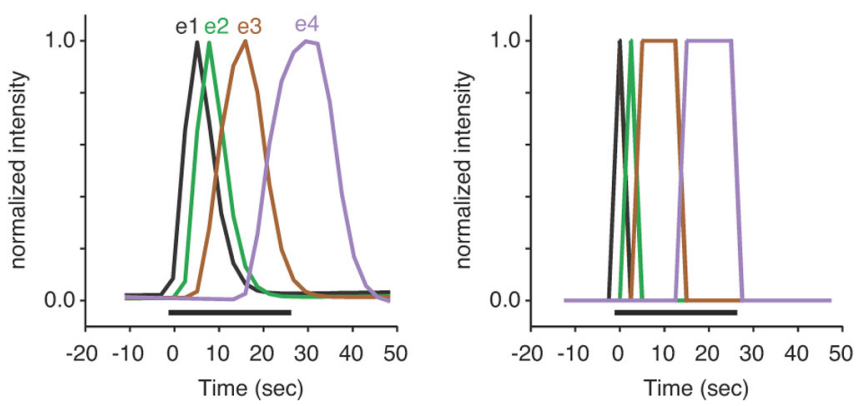

Figure 1. Derivation of hemodynamic response function (HRF) based on scout dataset ( $n=4$ subjects). The axial brain image shows significant activation of the scout dataset for the high painful heat condition $(Z>2.3$ [uncorrected for multiple comparisons]). Significant $\mathrm{S} 1$ activation in the scout dataset was identified by considering significant activation within left postcentral gyrus as identified by the probabilistic Harvard-0xford Cerebral Atlas at $>25 \%$ likelihood (green). A trial average response was calculated for voxels with significant contralateral S1 activation. Curve fitting divided the HRF EV into four parts (e1, e2, e3, and e4) using three splits points. The lower row shows the component EVs before and after gamma convolution. Note that e1 and e2 each represent adjacent and discrete samples in time, although they clearly formed a compound early phase in the HRF best fit. The set [e1, e2] was replaced with [e2, de2] to model the early phase. Refer to methods for details. A, Anterior; L, left; P, posterior; $R$, right.

intensity. To test this assertion, we used 3T fMRI to record BOLD responses to a range of innocuous and noxious temperatures in healthy volunteers. Perceptual ratings of heat intensity and pain intensity were collected for every temperature level to directly compare the relationship of these different sensory percepts with this measure of brain activity.

\section{Materials and Methods}

Subjects. Sixteen healthy right-handed subjects ( 8 males, 8 females; $28.8 \pm 7.7$ years old) were recruited by advertisement and provided written informed consent to participate in this experiment. All females reported contraceptive use, including oral contraception $(n=6)$, contraceptive intramuscular injection $(n=1)$, and a contraceptive vaginal ring $(n=1)$. Recent drug use was ruled out by negative results on a urine screen (Alfa Scientific Designs). The study was approved by the McLean Hospital Institutional Review Board, and met the scientific and ethical guidelines for human pain research of the Helsinki Accord (http://ohsr.od. nih.gov/guidelines/helsinki.html) and the International Association for the Study of Pain.

Thermal stimulation. A $1.6 \times 1.6 \mathrm{~cm}$ contact thermode (TSA-II, Medoc Advanced Medical Systems) was used to deliver thermal stimuli to the right cheek, in the area innervated by the maxillary division of the trigeminal nerve. More precisely, the thermode was fixed on the maxilla, just inferior and medial to the prominence of the zygomatic bone. The face was selected because of its high sensitivity to heat (Rolke et al., 2006). Six different target temperatures were used during fMRI scanning and were customized to each subject's perception as determined through prescan testing. The six selected stimulus levels represented a range of perceptual intensities, which included three innocuous and three painful temperatures based on subject reports (see Prescan testing, below, for details). At rest, the thermode maintained a steady baseline temperature of $35^{\circ} \mathrm{C}$. The target temperature was maintained for $24 \mathrm{~s}$, the rate of temperature change was $5^{\circ} \mathrm{C} / \mathrm{s}$, and the interstimulus interval was $30 \mathrm{~s}$.

Perceptual ratings. Subjects used computerized visual analog scales (VAS) to separately rate heat intensity and pain intensity using a hand-held analog dial in their left hand. The scales were presented horizontally, presented sequentially one after the other, and were titled "Heat" and "Pain." The markers for the extremes of the heat-intensity scale were labeled "No heat" to "Most intense," and "No pain" and "Most intense" for the pain intensity scale. The software package LabVIEW 5.1 (National Instruments) was used to display the scales. VAS ratings were recorded digitally as numerical values from $0-10$, although the scales did not display numbers to the subjects. For fMRI scans, ratings were collected after each scan.

Prescan testing. Subjects underwent quantitative sensory testing to determine the six temperatures that they were to receive during the fMRI scanning. An ascending staircase procedure was conducted, consisting of a resting baseline of $35^{\circ} \mathrm{C}$ and target temperatures that sequentially increased by $1^{\circ} \mathrm{C}$ at each step. Each temperature was delivered discretely, as described above in Thermal stimulation. Subjects rated heat and pain intensity after the completion of each stimulus. The six stimulus levels to be used in the scanner were selected in the following manner: (T1) low innocuous heatfirst nonzero rating of heat intensity (heat detection threshold); (T2) moderate innocuous heat-the midpoint between the low and high heat temperatures; (T3) high innocuous heat-the highest nonpainful temperature (pain detection threshold $-1^{\circ} \mathrm{C}$ ); (T4) low painful heat-first nonzero rating of pain intensity (pain detection threshold); T5) moderate painful heat - pain intensity VAS rating of " $4-6$ "; and T6) high painful heatpain intensity VAS rating of "7-10."

Scanning experimental paradigm. Each subject underwent a single scanning session, which included six randomized fMRI scans consisting of thermal stimulation presented in an event-related design. Each functional scan used a single target temperature that corresponded to one of the six different stimulus levels determined for each subject during prescan testing. fMRI scans consisted of a $42 \mathrm{~s}$ baseline period followed by three stimulus cycles, as described above. The high painful heat scan was always performed last, but the scan order for the other stimulus levels was randomized. After the completion of each fMRI scan, subjects retrospectively rated the average peak heat intensity and pain intensity experienced.

Image acquisition. Subjects were scanned in a 3T Siemens Trio MRI scanner using a circularly polarized head coil. Anatomical images were acquired using a magnetization prepared rapid gradient echo (MPRAGE) sequence (128 1.33-mm-thick slices with an in-plane resolution of $1 \mathrm{~mm}[256 \times 256])$. Functional scans were collected using an echo planar imaging $(\mathrm{EPI})$ sequence $(\mathrm{TE} / \mathrm{TR}=30 / 2500 \mathrm{~ms})$. Functional scans consisted of 41 slices, with $3.5 \mathrm{~mm}$ isometric resolution, with the acquisition coronally oriented to the match the brainstem axis and included the cerebellum. Eighty-eight volumes were captured in each fMRI $\operatorname{scan}(3: 40)$.

Individual subject level image preprocessing. Functional image datasets were processed and analyzed with FSL 4.1.5 (FMRIB's Software Library, www.fmrib.ox.ac.uk/fsl) (Smith et al., 2004). Visual screening of the functional volumes revealed that none of the subjects showed indications of gross movement ( $>1$ voxel). The initial two volumes were removed 
from each of the functional scans to allow for signal equilibration. The skull and other nonbrain areas were extracted from the anatomical and functional scans using the script brain extraction tool (BET) of FSL. Motion correction using FMRIB's Linear Image Registration Tool (MCFLIRT) was performed on each functional scan. All volumes were mean-based intensity normalized by the same factor. The volumes were spatially smoothed with a $5 \mathrm{~mm}$ full-width at half-maximum (FWHM) filter, and a $100 \mathrm{~s}$ high-pass temporal filter was applied.

Scout analysis to define appropriate hemodynamic response function. Previous imaging studies using contact thermodes suggest that the hemodynamic response for painful heat is biphasic, and is inadequately modeled using a conventional monophasic gamma-convolved explanatory variable (for review, see Upadhyay et al., 2010). The high painful heat scans from four randomly selected subjects served as a scout dataset to define the most appropriate hemodynamic response function for our experimental paradigm.

First-level fMRI analysis of single subject data was performed with FMRI Expert Analysis Tool using FMRIB's Improved Linear Model (FEAT FILM) Version 5.98 with local autocorrelation correction (Woolrich et al., 2001). For the scout dataset, the temperature profiles recorded during the high painful heat scan were rescaled from $0-1$ and entered as explanatory variables (EVs), as were their temporal derivatives to account for small temporal delays. Temperature EVs were convolved with a gamma function incorporating a $3 \mathrm{~s} \mathrm{SD}$ and a 6 s hemodynamic lag. Two additional covariates of no interest were included that modeled the mean signal time courses measured in white matter and CSF, as segmented by FMRIB's Automated Segmentation Tool (FAST). The resulting individual subject-level statistical maps from all FEAT analyses were coregistered with the subjects' anatomical images with FMRIB's Linear Image Registration Tool (FLIRT).

Group activation maps were generated by fMRI Expert Analysis Tool (FEAT) fMRIB's Local Analysis of Mixed Effects (FLAME). For the high painful heat scan, a fixed effects analysis was performed on the scout dataset (Fig. 1) to identify stimulus-related activation in contralateral primary somatosensory cortex (S1), as this region of interest (ROI) has previously shown biphasic responses to painful heat. Trial averaging in the scout subjects revealed two distinct positive responses in contralateral S1 [for methodological detail, refer to Moulton et al. (2007)].

An iterative procedure was performed to identify the most appropriate model to fit the observed left $\mathrm{S} 1$ trial average response. The single trial EV was divided into four parts (e1, e2, e3, e4) using three split points based on model fitting of the trial average (Fig. 1). For each choice of the 3 split points, the four parts e1, e2, e3, and e4 were as follows: demeaned, gamma convolved, demeaned, high-pass filtered (100 s), and demeaned. The convolved and high pass filtered e1, e2, e3, and e4 were entered into a general linear model (GLM) analysis to model the demeaned trial average response for left S1. A demeaned linear drift was added to the GLM as a confound of no interest to account for end effects in high-pass filtering. The mean squared error (MSE) of the GLM was saved as a model fit measure. We repeated this analysis for every possible combination of the three split points. Finally, we selected the split points that provided the smallest MSE over all combinations. The optimal split points were such that e1 corresponded to time point 6 , e2 to time point 7, e3 consisted of time points 8-11, and e4 consisted of time points 12-17. Note that a single time point separated e1 and e2. Hence, we replaced the set [e1, e2] by the set [e2, de2], where de2 is the temporal derivative of e2. The logic for doing this was to use e 2 to capture the "early" response and de2 to capture small variations in "onset" of the early response across subjects. We validated the selection of these split points by repeating the same analysis we performed for left S1 for two other functional ROIs (bilateral S1 and bilateral insula), which confirmed that the selected split points were near optimal for these additional ROIs as well. Thus, a 3 EV model was established that consisted of EV1 (e2, de2), EV2 (e3), and EV3 (e4).

Image analysis with the three-EV model. Image analysis for the experimental dataset of 12 subjects, not including the scout subjects, proceeded similarly as described above, but using the three-EV model at the single subject level. FLAME was run to perform two separate types of mixed effect group analyses, to detect the following: (1) high painful heat activation relating to each of the three EVs, and (2) BOLD correlation with
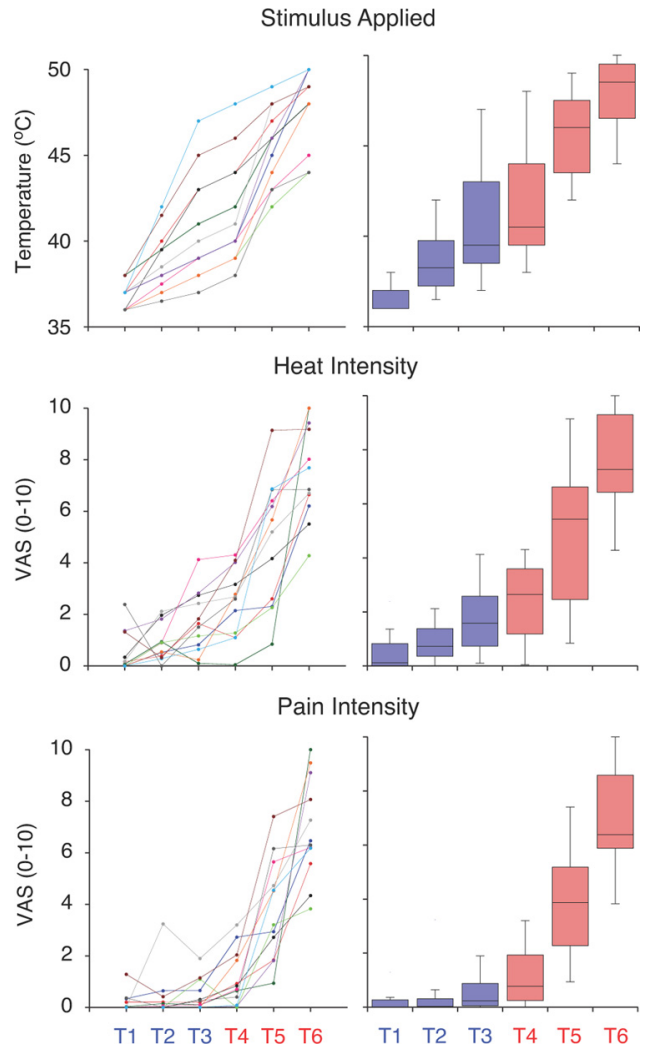

Figure 2. Psychophysical and stimulus data ( $n=12$ subjects). Perceptual ratings of heat intensity and pain intensity were statistically differentiable [two-way repeated-measures ANOVA (VAS modality, stimulus level), VAS modality $\left.F_{(1)}=6.367, p<0.05\right]$. Line graphs highlight within-subject differences in stimulus/ratings versus prescribed perceptual levels, with each line color representing a different subject. Line colors assigned to each subject are consistent across each line graph. Box plots highlight the median and distribution across subjects and were generated by the same data as their neighboring line graphs. The horizontal line within each box represents the median measurement, upper and bottom bounds of each box correspond to the upper and lower quartile range, and the whiskers indicate the complete range of samples. Box plots and prescribed perceptual levels are color-coded based on whether they are innocuous (blue) or painful (red).

Heat and Pain intensity ratings as parametric modulators entered into the same model, and a contrast of these correlational factors for each of the three EVs separately. For the correlation analysis, within-subject variation of heat and pain intensity ratings was taken into account. All activations, correlations, and contrasts had a significance threshold criterion of $Z>2.3$ with a cluster significance threshold of $p<0.05$ (corrected for multiple comparisons).

Statistical analysis of psychophysical measures. To test the differentiability of heat intensity and pain intensity reports, a two-way repeated-measures ANOVA was performed with the factors "VAS modality," consisting of two levels (Heat/Pain), and "stimulus level," consisting of six levels (T1/ $\mathrm{T} 2 / \mathrm{T} 3 / \mathrm{T} 4 / \mathrm{T} 5 / \mathrm{T} 6$ ). This was treated as a repeated-measures analysis, as all subjects rated Heat and Pain for each stimulus level, and all subjects received each of the six stimulus levels.

\section{Results}

\section{Psychophysics}

In the experimental dataset $(n=12)$, the temperatures that corresponded with the six stimulus levels were $36.8 \pm 0.8^{\circ} \mathrm{C}(\mathrm{T} 1)$, $38.8 \pm 1.8^{\circ} \mathrm{C}(\mathrm{T} 2), 40.8 \pm 3.1^{\circ} \mathrm{C}(\mathrm{T} 3), 41.8 \pm 3.1^{\circ} \mathrm{C}(\mathrm{T} 4), 45.6 \pm$ $2.2^{\circ} \mathrm{C}$ (T5), and $47.8 \pm 2.2^{\circ} \mathrm{C}$ (T6) (Fig. 2). Temperatures showed a significant increase with increasing stimulus level (repeatedmeasures ANOVA[stimulus level], $F_{(5)}=112.93, p<0.0001$ ).

The 12 subjects were able to differentiate between heat intensity and pain intensity across the range of stimulus levels tested in 


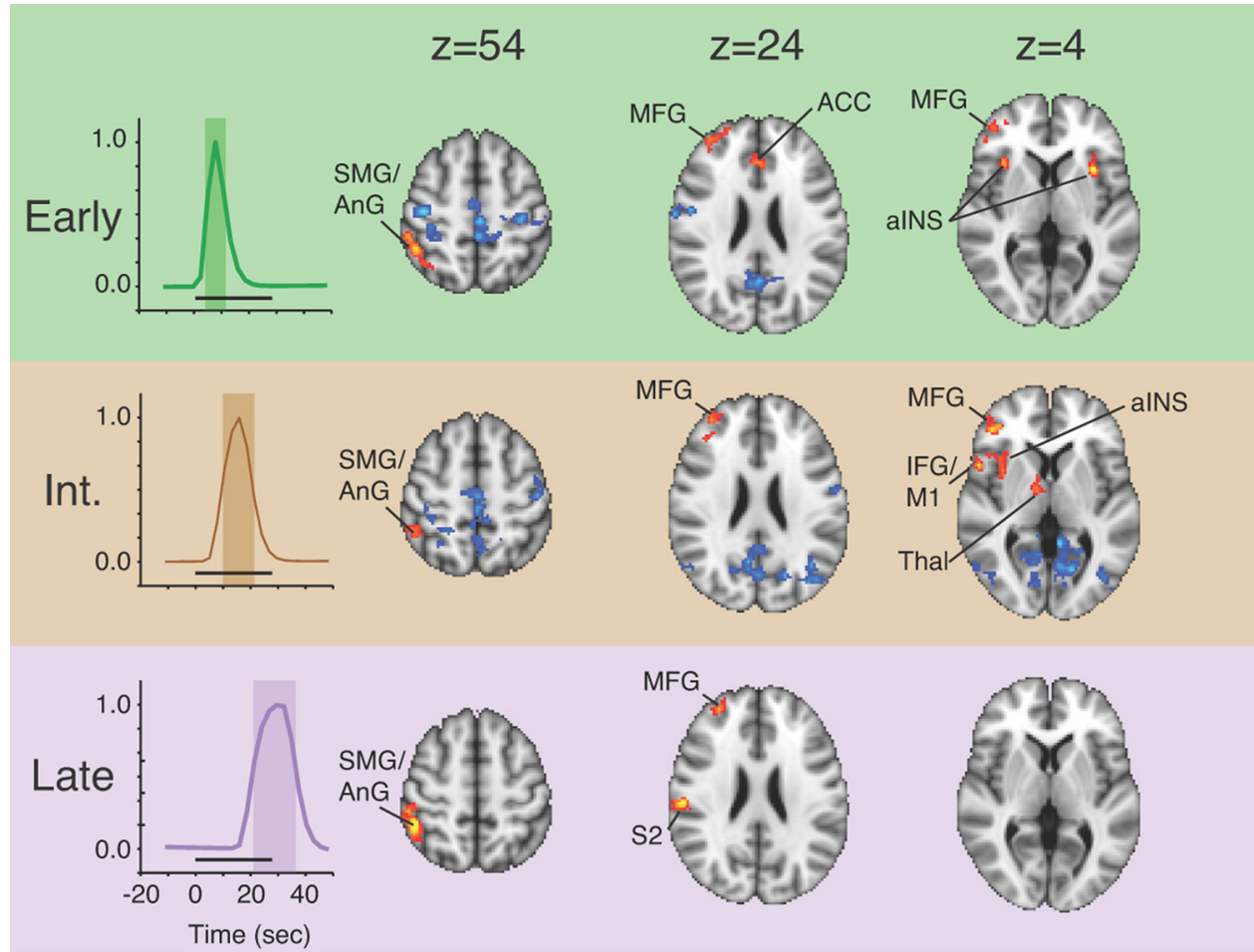

\section{Conventional EV}
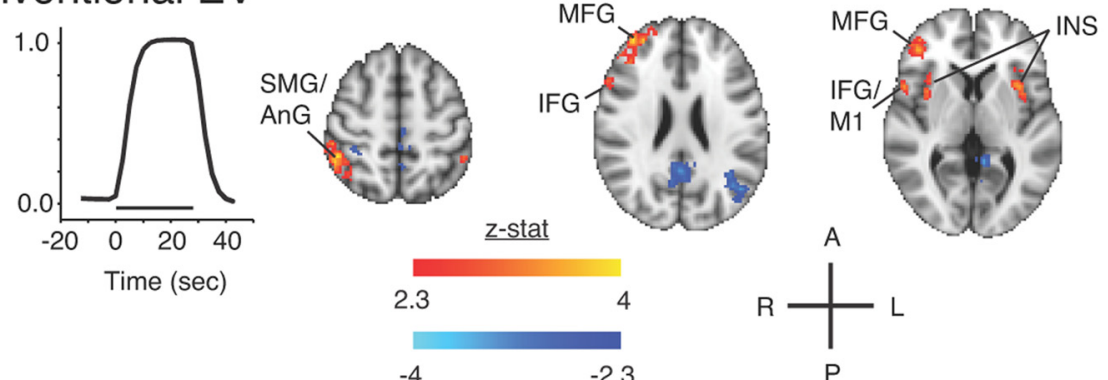

Figure 3. The temporal evolution of group activation to the high painful heat condition across the modeled HRF response. A conventional group analysis using a monophasic gamma-convolved EV for the same dataset is shown below for comparison. Significant activations were thresholded at $Z>2.3, p<0.05$ (cluster-corrected threshold). ACC, anterior cingulate cortex; AnG, angular gyrus; aINS, anterior insula; IFG, inferior frontal gyrus; INS, insula; M1, primary motor cortex; SMG, supramarginal gyrus; Thal, thalamus.

the scanner [Fig. 2, two-way repeated-measures ANOVA (VAS modality, stimulus level), VAS modality $F_{(1)}=6.367, p<0.05$ ]. A significant effect of stimulus level on perceptual ratings was also observed [Fig. 2, two-way repeated-measures ANOVA (VAS modality, stimulus level), stimulus level $F_{(5)}=109.132, p<0.0001$ ]. For perceptual ratings, no significant interaction between VAS modality and stimulus level was detected [two-way repeatedmeasures ANOVA (VAS modality, stimulus level), interaction $\left.F_{(5)}=0.584, p=0.71\right]$.

\section{High painful heat group-level activation}

Different patterns of regional brain activation were detected for each of the three EVs corresponding to "early phase," "interval phase," and "late phase" responses (Fig. 3; Table 1). The early phase EV detected significant activation in midline anterior cingulate cortex, bilaterally in anterior insula, and ipsilaterally in supramarginal gyrus, angular gyrus, superior parietal lobule, and middle frontal gyrus. The interval phase EV also detected activation ipsilaterally in anterior insula, supramarginal gyrus, angular gyrus, superior parietal lobule, and middle frontal gyrus, but additional activation was found ipsilaterally in thalamus, inferior frontal gyrus, and primary motor cortex. Activations in the anterior cingulate cortex and contralateral anterior insula, found in the early phase, were not detected during the interval phase. Similar to the early and interval phases, the late phase EV detected activation ipsilaterally in supramarginal gyrus, angular gyrus, superior parietal gyrus, and middle frontal gyrus. The late phase EV also revealed novel prominent activation in ipsilateral secondary somatosensory cortex (S2). Both early and late phase EVs detected activation in contralateral primary somatosensory cortex for $Z>2.3$, but only when not applying the cluster significance threshold.

\section{Brain activations correlated with perceptual intensity}

Only late phase EV-related brain activity showed significant correlations with perceptual ratings for heat and pain (Fig. 4; Table $2)$. Heat VAS ratings were significantly correlated with contralateral S1, S2, primary motor cortex, and superior/middle temporal gyrus. Pain VAS ratings were significantly correlated with bilateral thalamus, in the vicinity of the ventroposteriomedial thalamus. A contrast of Heat VAS- and Pain VAS-correlated activity revealed a significantly greater slope for the BOLD-VAS correla- 
Table 1. High painful heat activation detected by the three $\mathrm{EV}$ model $(\mathrm{Z}>2.3, p<$ 0.05 [cluster-corrected threshold])

\begin{tabular}{lcll}
\hline Cluster* & $\begin{array}{l}\text { MNI }\left(Z_{\max }\right) \\
x_{1}, y, z\end{array}$ & $\begin{array}{l}\text { Max } \\
Z \text {-stat }\end{array}$ & $\begin{array}{l}\text { Vol } \\
\left(\mathrm{cm}^{3}\right.\end{array}$ \\
\hline Early & & & \\
$\quad$ R FrOrb/alNS/TmP & $42,18,-10$ & 4.48 & 4.37 \\
$\quad$ LalNS/FrOp & $-32,14,2$ & 4.41 & 2.67 \\
R FrPole & $38,52,10$ & 3.34 & 2.55 \\
AnG/SMG/SPL & $46,-48,50$ & 4.13 & 2.45 \\
R ParaCG/ACC & $4,28,34$ & 3.77 & 2.16 \\
Interval & & & \\
R FrOp/aINS/IFG/COp/PrCG/TmP & $48,18,-6$ & 4.01 & 4.25 \\
R SMG/AnG/SPL & $52,-34,40$ & 3.84 & 4.15 \\
R FrPole & $40,40,2$ & 4.51 & 3.51 \\
R Thal & $14,-4,14$ & 3.36 & 1.86 \\
Late & & & \\
R SMG/AnG/SPL/Pa0p/LOC & $48,-44,54$ & 4.94 & 6.74 \\
R FrPole & $30,52,22$ & 3.83 & 1.90 \\
\hline
\end{tabular}

* Region with maximum Z statistic appears first. ACC, Anterior cingulate cortex; AnG, angular gyrus; alNS, anterior insula; $\mathrm{COp}$, central opercular cortex; FrOp, frontal operculum cortex; FrOrb, frontal orbital cortex; FrPole, frontal pole; IFG, inferior frontal gyrus; LOc, lateral occipital cortex; PTm, planum temporale; Pa0p, parietal operculum cortex; ParaCG, paracingulate gyrus; PrCG, precentral gyrus; SMG, supramarginal gyrus; SPL, superior parietal lobule; STG, superior temporal gyrus; Thal, thalamus; TmP, temporal pole; R, right; L, left; Max, maximum; Vol, volume; stat, statistic.

tion for Heat ratings in contralateral primary and secondary somatosensory cortices, primary motor cortex, and superior/ middle temporal gyrus. The same contrast revealed no areas with significantly increased slope for the BOLD-VAS relationship for Pain ratings. An identical control analysis for these contrasts that only included scans with pain reports above zero ( $n=56$ of72 scans) revealed the same outcome as observed in the full analysis (data not shown). Thus, the main result does not reflect a difference in the numbers of nonzero values rather than a true physiological difference.

\section{Discussion}

Across a range of innocuous and noxious temperatures, BOLD signals in S1 and S2 contralateral to stimulation were statistically better correlated with perceptual ratings of heat intensity than pain intensity. Our results suggest that fMRI measures of neural activity in somatosensory cortices better reflect magnitude of heat sensation, rather than pain intensity specifically. These correlational differences, as well as the correlations themselves, were specific only to late phase responses. Also, early, interval, and late phase analyses revealed different subsets of brain activation related to noxious heat. These findings suggest a temporal differentiation of brain responses to heat, which may indicate activation of different functional networks.

\section{Heat intensity encoding}

That heat sensation is more closely related to activity in primary somatosensory cortex than pain sensation is alluded to by animal research. Electrophysiological recordings in primate S1 indicate that neurons that respond to a range of heat (wide dynamic range) fire more vigorously than those that respond specifically to noxious heat (nociceptive specific) (Kenshalo et al., 2000). Anesthesia, usually in combination with an agent inducing muscle paralysis, does not eliminate $S 1$ responses to acute noxious heat measured electrophysiologically in primates (Kenshalo and Isensee, 1983; Chudler et al., 1990; Kenshalo et al., 2000). Although diminished, persistent responses to noxious thermal stimuli suggest that neural activity in S1 does not rely on conscious perception and may better reflect the neural representation of heat intensity rather than pain. In turn, perhaps separate percepts of heat and pain arise from the evaluation of how S1 encodes heat intensity. Notably, neuroimaging studies in rodents suggest that other painful stimuli aside from heat also show persistent responses in somatosensory cortex and other regions, even under anesthesia (for review, see Borsook and Becerra, 2011).

Although animal literature indicates that S2 neurons responsive to noxious thermal stimuli are rare (Robinson and Burton, 1980; Dong et al., 1994), S2 has been specifically implicated in heat-intensity coding in electrophysiological recordings in humans. Electroencephalography in healthy subjects has related intensity coding of noxious laser stimuli to evoked potential amplitude in operculoinsular region, as well as S1 (Iannetti et al., 2005). Intracortical recordings of laser-evoked potentials in patients indicated that $\mathrm{S} 2$ is capable of encoding fine discrimination of both innocuous and painful heat intensity (Frot et al., 2007). The correlation we observed between heat-intensity ratings and BOLD responses in S2 suggests that $\mathrm{AMRI}$ is also capable of detecting heat intensity-dependent S2 responses previously measured with intracortical electrodes.

\section{Pain intensity coding?}

Our analysis did not detect any brain responses with a statistically greater correlation with pain intensity ratings than heat-intensity ratings. As heat-intensity perception was well correlated with activity in somatosensory cortices, this suggests that pain may arise secondarily to the primary sensation of heat through a cognitive/ evaluative process. In other words, S1 and S2 encoded the intensity of the heat stimulus, heat perception closely mirrored this cortical representation of heat intensity, and multimodal evaluative brain regions interpreted this heat representation in terms of its level of painfulness.

Candidate areas for this cognitive evaluative process may include right dorsolateral prefrontal cortex, supramarginal gyrus, and angular gyrus, which were the only brain regions persistently active throughout T6. A similar evaluative role for right prefrontal cortex has been suggested previously for heat pain (Coghill et al., 1999; Kong et al., 2006), and other evidence suggests that a multisensory magnitude estimation process may occur in lateral prefrontal cortex and adjoining insula (Baliki et al., 2009). Although right supramarginal gyrus and angular gyrus have not been explicitly linked to evaluative processes, these parietal areas have been implicated with multisensory spatial processing (Calvert, 2001; Renier et al., 2009) and, when damaged, sensory neglect (Mesulam, 1981; Mort et al., 2003). Activation of these multisensory processing regions during heat stimuli likely reflects attentional processes that are automatically engaged (Derbyshire et al., 1997; Coghill et al., 2001), which may play a further role in cognitive evaluation of such stimuli. An alternative explanation is that evaluation of stimuli represented in somatosensory cortices as painful is a distributed process, and occurs over a network of areas activated with noxious stimuli (Coghill et al., 1994; Apkarian et al., 2005; Kong et al., 2006; Seminowicz and Davis, 2006; Helmchen et al., 2008).

\section{Temporal differentiation of activation "networks"}

By differentiating three distinct phases of the hemodynamic response, we were able to detect changes in brain activation patterns over the course of stimulus application. Previous time course investigations of BOLD responses to noxious contact heat have reported a stereotypical biphasic response (Becerra et al., 2001; Wager et al., 2004; Upadhyay et al., 2010), which appear to share the same temporal profile as we recorded in our scout da- 

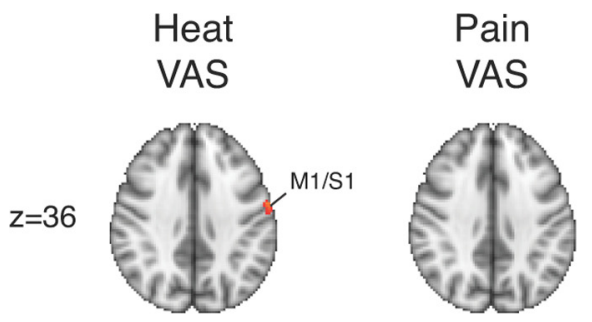

Heat-Pain
VAS
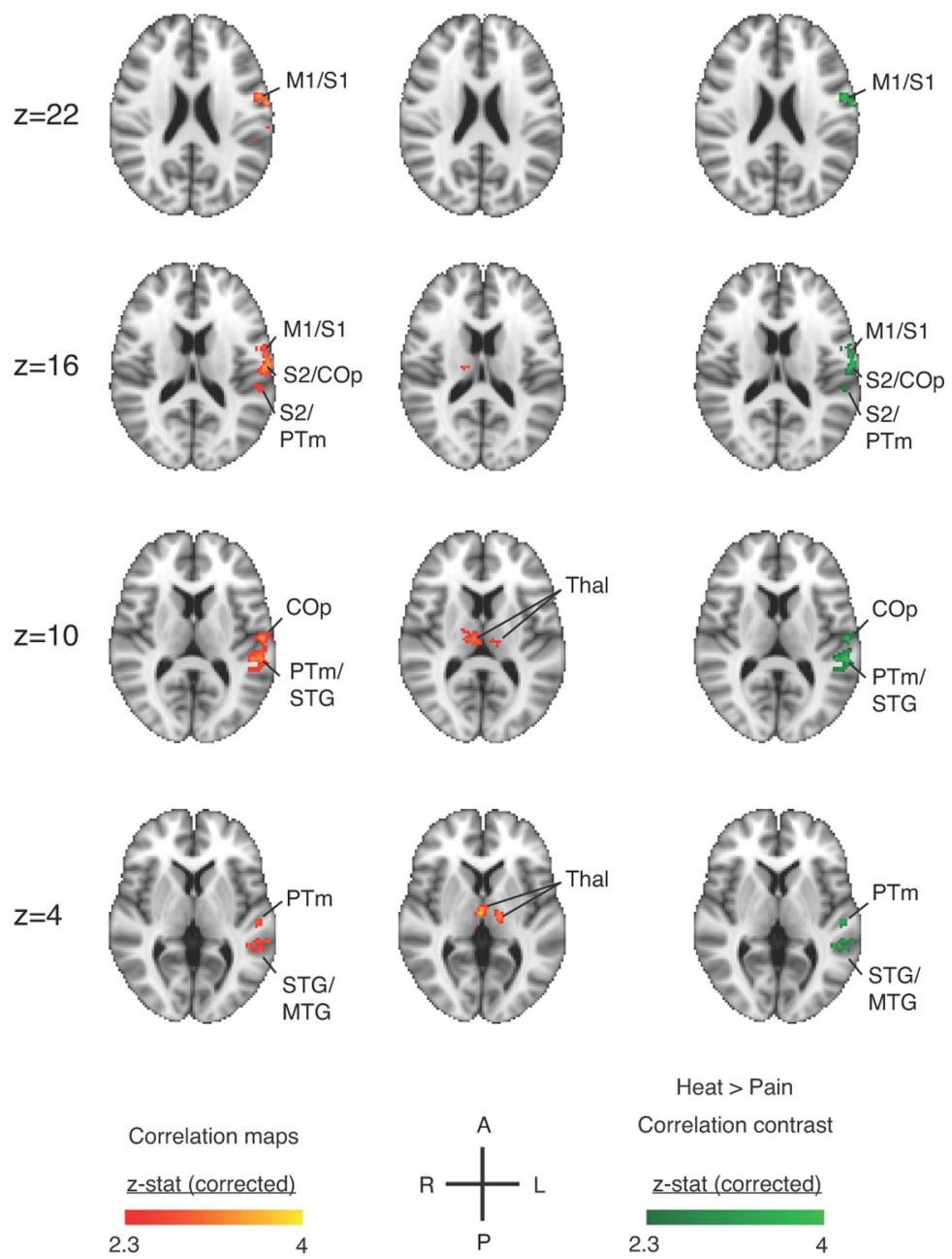

Figure 4. Perceptual ratings correlate with late phase responses across all scans for the six perceptual levels ( $n=12$ subjects, 72 scans). Early and interval phases exhibited no significant correlations with heat or pain ratings. The general linear model used regressors for heat and pain intensity ratings in the same model and accounted for within-subject variation. Significant correlations to heat ratings were detected in the somatosensory cortices contralateral to stimulus application. Significant correlations to ratings are colored red-yellow $[Z>2.3, p<0.05$ (cluster-corrected threshold)], and significant contrasts for Heat $>$ Pain correlations are green $[Z>2.3, p<0.05$ (cluster-corrected threshold)]. No significant contrast was observed for Pain $>$ Heat correlations. COp, central opercular cortex; M1, primary motor cortex; MTG, middle temporal gyrus; PTm, planum temporale; PoCG, postcentral gyrus; PrCG, precentral gyrus; SMG, supramarginal gyrus; STG, superior temporal gyrus; Thal, thalamus.

taset. Previously, the biphasic response was defined by dividing the response into two halves, whereas we found that curve fitting of the response in contralateral S1 modeled early and late phases separated by an intermediate interval phase. Different patterns of brain activity associated with these three phases, in addition to previous reports of biphasic responses, suggest a temporal differentiation of functional networks activated during noxious contact heat. This "biphasic" hemodynamic response with distinct early, interval, and late phases may be particular for noxious contact heat, as responses to brief noxious laser stimuli are monophasic (Bornhövd et al., 2002; Büchel et al., 2002). Long duration contact heat would result in more sustained drive of primary afferents, as well as dynamic changes in attention, endogenous pain modulation, and cognitive valuation, all of which could potentially contribute to the biphasic response we observed. Thus, our results may apply specifically to contact heat stimulation as used in this study.

The early phase featured prominent activation of anterior cingulate cortex and bilateral anterior insula. These areas are consistently tied to pain processing, although specificity of these responses to pain is somewhat controversial (Legrain et al., 2011). A recent fMRI study found that cingulate and insular responses to painful laser stimuli could reflect a multimodal salience-detection process (Mouraux et al., 2011). Activation during early phase has also been suggested to reflect an early threat-detection process and autonomic affective valuation of aversive stimuli (Becerra et al., 2001; Maihöfner et al., 2011), and our finding that cingulate and insula activate during early phase is also consistent with their involvement in transient attention orienting processes (Davis et al., 1997; Peyron et al., 1999; Wiech et al., 2010; Mouraux et al., 2011). Perhaps monophasic responses to extremely brief laser stimuli (Bornhövd et al., 2002; Büchel et al., 2002) and innocuous contact heat (Moulton et al., 2005) reflect an isolated triggering of this early phase that relates to stimulus detection.

The late phase featured activation of ipsilateral S2, as well as heat intensitycorrelated brain activity in contralateral S1 and S2. This late phase was the only part of the BOLD response to heat that had significant correlations with perceptual ratings. These data are consistent with previous findings that the late phase corresponded with: (1) fine discrimination of noxious heat intensity in contralateral S1 (Moulton et al., 2005); (2) the conscious CNS response to pain (Becerra et al., 2001); (3) the gradual ascent of online pain intensity ratings that correlated with BOLD responses to noxious heat in contralateral S1 and S2 (Chen et al., 2002); (4) optical imaging in squirrel monkeys that revealed temporal summation of long latency noxious responses in S1 that correlated with human reports of pain intensity to similar heat stimuli (Tommerdahl et al., 1996); and (5) decreases with reduced pain from contact heat during placebo analgesia (Wager et al., 2004). Together, this evidence indicates that the late phase is related to perceptual intensity of noxious stimuli.

\section{Caveats}

BOLD responses in other brain regions previously shown to correlate with pain intensity, such as parts of insula and anterior 


\begin{tabular}{|c|c|c|c|}
\hline Cluster* $^{*}$ & $\begin{array}{l}\operatorname{MNI}\left(z_{\max }\right) \\
x, y, z\end{array}$ & $\begin{array}{l}\text { Max } \\
\text { Z-stat }\end{array}$ & $\begin{array}{l}\text { Vol } \\
\left(\mathrm{cm}^{3}\right.\end{array}$ \\
\hline \multicolumn{4}{|l|}{ Heat VAS correlation } \\
\hline L PrCG/PoCG/COp/PTm/STG/MTG & $-56,0,20$ & 3.88 & 6.15 \\
\hline \multicolumn{4}{|l|}{ Pain VAS correlation } \\
\hline R Thal/L Thal & $2,-14,4$ & 3.72 & 2.26 \\
\hline \multicolumn{4}{|l|}{ Heat VAS versus Pain VAS correlations } \\
\hline L PrCG/PoCG/COp/PTm/STG/MTG & $-56,0,20$ & 3.86 & 5.62 \\
\hline L PrCG/R PrCG/R PoCG & $-14,-22,66$ & 3.98 & 2.37 \\
\hline
\end{tabular}

*Region with maximum Z statistic appears first. PoCG, postcentral gyrus; COp, central opercular; MTG, middle temporal gyrus; PTm, planum temporale; PoCG, postcentral gyrus; $\operatorname{PrCG}$, precentral gyrus; SMG, supramarginal gyrus; STG, superior temporal gyrus; Thal, thalamus; R, right; L, left; Max, maximum; Vol, volume; stat, statistic.

cingulate cortex, were not significantly related to either Heat or Pain VAS reports. A likely possibility is that nuisance covariates, which modeled signals within white matter and CSF, may have captured some variance associated with stimulus-related brain activity. This was found to be the case in our dataset (data not shown). These nuisance covariates thus resulted in a conservative estimate for areas that are involved in Heat and Pain intensity coding. Note that we focused on detecting differences between Heat and Pain correlations, and do not contest the correlation between brain activity and pain intensity.

While our results appear to indicate that perception of pain may (1) arise from cognitive valuation of stimulus properties such as heat, or (2) reflect the cumulative effect of several distributed processes, other nuanced interpretations are also possible. Previous studies in patients have shown that direct electrical stimulation of the insula and S2 can produce pain (Mazzola et al., 2011). Thus, pain can arise from discrete, localized areas of neural activity, although it may not be possible to cleanly separate pain from heat sensation in this manner. Also, patients with lesions incorporating posterior insula and S2 have shown impaired pain and temperature detection (Greenspan et al., 1999), indicating either disruption of serial processing of stimulus encoding or colocalization of function. Another possibility is that pain reports may not correlate as well with BOLD responses simply because brain activity may be a purer measure of heat and/or nociceptive signals than perceptual reports of pain, which are influenced by cognitive and evaluative processes. And finally, the relationship between Heat versus Pain reports and brain activity may reflect different profiles of nonlinearity of the two percepts with the BOLD signal itself. Clearly, further study is required to address such issues.

\section{Conclusions}

This study indicates that late phase BOLD responses to contact heat in contralateral S1 and S2 are significantly better correlated with heat intensity than pain intensity. Dividing hemodynamic responses into early, interval, and late revealed different patterns of brain activation that may reflect different aspects of the experience of pain and stimulus processing (i.e., salience/affect, cognitive/evaluative, and sensory/discriminative). Our results urge caution when interpreting BOLD responses elicited by painful stimuli as "pain processing," especially when pain is associated with other perceived stimulus properties.

\section{References}

Apkarian AV, Bushnell MC, Treede RD, Zubieta JK (2005) Human brain mechanisms of pain perception and regulation in health and disease. Eur J Pain 9:463-484.
Baliki MN, Geha PY, Apkarian AV (2009) Parsing pain perception between nociceptive representation and magnitude estimation. J Neurophysiol 101:875-887.

Becerra L, Breiter HC, Wise R, Gonzalez RG, Borsook D (2001) Reward circuitry activation by noxious thermal stimuli. Neuron 32:927-946.

Bornhövd K, Quante M, Glauche V, Bromm B, Weiller C, Büchel C (2002) Painful stimuli evoke different stimulus-response functions in the amygdala, prefrontal, insula and somatosensory cortex: a single-trial fMRI study. Brain 125:1326-1336.

Borsook D, Becerra L (2011) CNS animal fMRI in pain and analgesia. Neurosci Biobehav Rev 35:1125-1143.

Büchel C, Bornhovd K, Quante M, Glauche V, Bromm B, Weiller C (2002) Dissociable neural responses related to pain intensity, stimulus intensity, and stimulus awareness within the anterior cingulate cortex: a parametric single-trial laser functional magnetic resonance imaging study. J Neurosci 22:970-976.

Bushnell MC, Duncan GH, Hofbauer RK, Ha B, Chen JI, Carrier B (1999) Pain perception: is there a role for primary somatosensory cortex? Proc Natl Acad Sci U S A 96:7705-7709.

Calvert GA (2001) Crossmodal processing in the human brain: insights from functional neuroimaging studies. Cereb Cortex 11:1110-1123.

Chen JI, Ha B, Bushnell MC, Pike B, Duncan GH (2002) Differentiating noxious- and innocuous-related activation of human somatosensory cortices using temporal analysis of fMRI. J Neurophysiol 88:464-474.

Chudler EH, Anton F, Dubner R, Kenshalo DR Jr (1990) Responses of nociceptive SI neurons in monkeys and pain sensation in humans elicited by noxious thermal stimulation: effect of interstimulus interval. J Neurophysiol 63:559-569.

Coghill RC, Talbot JD, Evans AC, Meyer E, Gjedde A, Bushnell MC, Duncan GH (1994) Distributed processing of pain and vibration by the human brain. J Neurosci 14:4095-4108.

Coghill RC, Sang CN, Maisog JM, Iadarola MJ (1999) Pain intensity processing within the human brain: a bilateral, distributed mechanism. J Neurophysiol 82:1934-1943.

Coghill RC, Gilron I, Iadarola MJ (2001) Hemispheric lateralization of somatosensory processing. J Neurophysiol 85:2602-2612.

Craig AD, Chen K, Bandy D, Reiman EM (2000) Thermosensory activation of insular cortex. Nat Neurosci 3:184-190.

Davis KD, Taylor SJ, Crawley AP, Wood ML, Mikulis DJ (1997) Functional MRI of pain- and attention-related activations in the human cingulate cortex. J Neurophysiol 77:3370-3380.

Derbyshire SW, Jones AK, Gyulai F, Clark S, Townsend D, Firestone LL (1997) Pain processing during three levels of noxious stimulation produces differential patterns of central activity. Pain 73:431-445.

Dong WK, Chudler EH, Sugiyama K, Roberts VJ, Hayashi T (1994) Somatosensory, multisensory, and task-related neurons in cortical area $7 \mathrm{~b}(\mathrm{PF})$ of unanesthetized monkeys. J Neurophysiol 72:542-564.

Frot M, Magnin M, Mauguière F, Garcia-Larrea L (2007) Human SII and posterior insula differently encode thermal laser stimuli. Cereb Cortex 17:610-620.

Greenspan JD, Lee RR, Lenz FA (1999) Pain sensitivity alterations as a function of lesion location in the parasylvian cortex. Pain 81:273-282.

Helmchen C, Mohr C, Roehl M, Bingel U, Lorenz J, Büchel C (2008) Common neural systems for contact heat and laser pain stimulation reveal higher-level pain processing. Hum Brain Mapp 29:1080-1091.

Iannetti GD, Zambreanu L, Cruccu G, Tracey I (2005) Operculoinsular cortex encodes pain intensity at the earliest stages of cortical processing as indicated by amplitude of laser-evoked potentials in humans. Neuroscience 131:199-208.

Johnstone T, Salomons TV, Backonja MM, Davidson RJ (2012) Turning on the alarm: The neural mechanisms of the transition from innocuous to painful sensation. Neuroimage 59:1594-1601.

Kenshalo DR Jr, Isensee O (1983) Responses of primate SI cortical neurons to noxious stimuli. J Neurophysiol 50:1479-1496.

Kenshalo DR, Iwata K, Sholas M, Thomas DA (2000) Response properties and organization of nociceptive neurons in area 1 of monkey primary somatosensory cortex. J Neurophysiol 84:719-729.

Kong J, White NS, Kwong KK, Vangel MG, Rosman IS, Gracely RH, Gollub RL (2006) Using fMRI to dissociate sensory encoding from cognitive evaluation of heat pain intensity. Hum Brain Mapp 27:715-721.

Legrain V, Iannetti GD, Plaghki L, Mouraux A (2011) The pain matrix re- 
loaded: a salience detection system for the body. Prog Neurobiol 93:111-124.

Maihöfner C, Seifert F, Decol R (2011) Activation of central sympathetic networks during innocuous and noxious somatosensory stimulation. Neuroimage 55:216-224.

Mazzola L, Isnard J, Peyron R, Mauguiere F (2011) Stimulation of the human cortex and the experience of pain: Wilder Penfield's observations revisited. Brain 135 [Pt 2]:631-640.

Mesulam MM (1981) A cortical network for directed attention and unilateral neglect. Ann Neurol 10:309-325.

Mort DJ, Malhotra P, Mannan SK, Rorden C, Pambakian A, Kennard C, Husain M (2003) The anatomy of visual neglect. Brain 126:1986-1997.

Moulton EA, Keaser ML, Gullapalli RP, Greenspan JD (2005) Regional intensive and temporal patterns of functional MRI activation distinguishing noxious and innocuous contact heat. J Neurophysiol 93:2183-2193.

Moulton EA, Pendse G, Morris S, Strassman A, Aiello-Lammens M, Becerra L, Borsook D (2007) Capsaicin-induced thermal hyperalgesia and sensitization in the human trigeminal nociceptive pathway: an fMRI study. Neuroimage 35:1586-1600.

Mouraux A, Diukova A, Lee MC, Wise RG, Iannetti GD (2011) A multisensory investigation of the functional significance of the "pain matrix." Neuroimage 54:2237-2249.

Olausson H, Charron J, Marchand S, Villemure C, Strigo IA, Bushnell MC (2005) Feelings of warmth correlate with neural activity in right anterior insular cortex. Neurosci Lett 389:1-5.

Peyron R, Garcia-Larrea L, Gregoire MC, Costes N, Convers P, Lavenne F, Mauguiere F, Michel D, Laurent B (1999) Haemodynamic brain responses to acute pain in humans: sensory and attentional networks. Brain 122 [Pt 9]:1765-1780.

Porro CA, Cettolo V, Francescato MP, Baraldi P (2003) Functional activity mapping of the mesial hemispheric wall during anticipation of pain. Neuroimage 19:1738-1747.

Renier LA, Anurova I, De Volder AG, Carlson S, VanMeter J, Rauschecker JP (2009) Multisensory integration of sounds and vibrotactile stimuli in processing streams for "what" and "where." J Neurosci 29:10950-10960.
Ringler R, Greiner M, Kohlloeffel L, Handwerker HO, Forster C (2003) BOLD effects in different areas of the cerebral cortex during painful mechanical stimulation. Pain 105:445-453.

Robinson CJ, Burton H (1980) Somatic submodality distribution within the second somatosensory (SII), 7b, retroinsular, postauditory, and granular insular cortical areas of M. fascicularis. J Comp Neurol 192:93-108.

Rolke R, Baron R, Maier C, Tölle TR, Treede RD, Beyer A, Binder A, Birbaumer N, Birklein F, Bötefür IC, Braune S, Flor H, Huge V, Klug R, Landwehrmeyer GB, Magerl W, Maihöfner C, Rolko C, Schaub C, Scherens A, et al. (2006) Quantitative sensory testing in the German Research Network on Neuropathic Pain (DFNS): standardized protocol and reference values. Pain 123:231-243.

Seminowicz DA, Davis KD (2006) Cortical responses to pain in healthy individuals depends on pain catastrophizing. Pain 120:297-306.

Smith SM, Jenkinson M, Woolrich MW, Beckmann CF, Behrens TE, Johansen-Berg H, Bannister PR, De Luca M, Drobnjak I, Flitney DE, Niazy RK, Saunders J, Vickers J, Zhang Y, De Stefano N, Brady JM, Matthews PM (2004) Advances in functional and structural MR image analysis and implementation as FSL. Neuroimage 23 [Suppl 1]:S208-S219.

Tommerdahl M, Delemos KA, Vierck CJ Jr, Favorov OV, Whitsel BL (1996) Anterior parietal cortical response to tactile and skin-heating stimuli applied to the same skin site. J Neurophysiol 75:2662-2670.

Upadhyay J, Pendse G, Anderson J, Schwarz AJ, Baumgartner R, Coimbra A, Bishop J, Knudsen J, George E, Grachev I, Iyengar S, Bleakman D, Hargreaves R, Borsook D, Becerra L (2010) Improved characterization of BOLD responses for evoked sensory stimuli. Neuroimage 49:2275-2286.

Wager TD, Rilling JK, Smith EE, Sokolik A, Casey KL, Davidson RJ, Kosslyn SM, Rose RM, Cohen JD (2004) Placebo-induced changes in FMRI in the anticipation and experience of pain. Science 303:1162-1167.

Wiech K, Lin CS, Brodersen KH, Bingel U, Ploner M, Tracey I (2010) Anterior insula integrates information about salience into perceptual decisions about pain. J Neurosci 30:16324-16331.

Woolrich MW, Ripley BD, Brady M, Smith SM (2001) Temporal autocorrelation in univariate linear modeling of FMRI data. Neuroimage 14: $1370-1386$ 